\title{
EUCLID'S ALGORITHM IN CYCLOTOMIC FIELDS
}

\author{
H. W. LENSTRA, JR.
}

\section{Introduction}

For a positive integer $m$, let $\zeta_{m}$ denote a primitive $m$-th root of unity. By $\phi$ we mean the Euler $\phi$-function. In this paper we prove the following theorem.

THEOREM. Let $\phi(m) \leqslant 10, m \neq 16, m \neq 24$. Then $\mathbf{Z}\left[\zeta_{m}\right]$ is Euclidean for the usual norm map.

Since $\mathbb{Z}\left[\zeta_{m}\right]=\mathbb{Z}\left[\zeta_{2 m}\right]$ for $m$ odd, this gives eleven non-isomorphic Euclidean rings, corresponding to $m=1,3,4,5,7,8,9,11,12,15,20$. The cases $m=1,3,4$, $5,8,12$ are more or less" classical [2 (pp. 117-118 and pp. 391-393); 8 ; 5 (pp. 228-231); 3 (chapters 12,14 and 15); 4; 7]. The other five cases are apparently new.

For $m$ even, the ring $\mathbb{Z}\left[\zeta_{m}\right]$ has class number one if and only if $\phi(m) \leqslant 20$ or $m=70,84$ or 90 , see [6]. So there are exactly thirty non-isomorphic rings $\mathbb{Z}\left[\zeta_{m}\right]$ which admit unique factorization. If certain generalized Riemann hypotheses would hold, then all these thirty rings would be Euclidean for some function different from the norm map [9].

\section{The general measure and Euclid's algorithm}

In this section $K$ denotes an algebraic number field of finite degree $d$ over $\mathbf{Q}$, and $K_{\mathbf{R}}$ is the $\mathbf{R}$-algebra $K \otimes_{\mathbf{Q}} \mathbf{R}$. Following Gauss $[\mathbf{2} ;$ p. 395] we define the general measure $\mu: K_{\mathbf{R}} \rightarrow \mathbf{R}$ by

$$
\mu(x)=\sum_{\sigma}|\sigma(x)|^{2}, \quad \text { for } \quad x \in K_{\mathbf{R}},
$$

the sum ranging over the $d$ different $\mathbf{R}$-algebra homomorphisms $\sigma: K_{\mathbf{R}} \rightarrow \mathbf{C},(c f .[1])$. It is easily seen that $\mu$ is a positive definite quadratic form on the $\mathbf{R}$-vector space $K_{\mathbf{R}}$.

Let $R$ be a subring of $K$ which is integral over $\mathbf{Z}$ and has $K$ as its field of fractions. Then $R$ is a lattice of maximal rank $d$ in $K_{\mathbf{R}}$. The fundamental domain $F$ with respect to $R$ is defined by

$$
F=\left\{x \in K_{\mathbf{R}} \mid \mu(x) \leqslant \mu(x-y) \text { for all } y \in R\right\} .
$$

This is a compact subset of $K_{\mathbf{R}}$ which satisfies

$$
F+R=K_{\mathbf{R}}
$$

Let

$$
c=\max \{\mu(x) \mid x \in F\} .
$$

A real number $c^{\prime}$ is called a bound for $F$ if $c^{\prime} \geqslant c$. A bound $c^{\prime}$ for $F$ is usable if for every $x \in F \cap K$ satisfying $\mu(x)=c^{\prime}$ there is a root of unity $u \in R$ such that $\mu(x-u)=c^{\prime}$. Note that every real number $c^{\prime}>c$ is a usable bound, since no $x \in F$ satisfies $\mu(x)=c^{\prime}>c$.

Received 14 May, 1974.

[J. LONDON MATH. Soc. (2), 10 (1975), 457-465] 
The norm $N: K_{\mathbf{R}} \rightarrow \mathbf{R}$ is defined by

$$
N(x)=\prod_{\sigma}|\sigma(x)|, \quad \text { for } \quad x \in K_{\mathbf{R}},
$$

the product ranging over the $\mathbf{R}$-algebra homomorphisms $\sigma: K_{\mathbf{R}} \rightarrow \mathbf{C}$. The arithmeticgeometric mean inequality implies

$$
N(x)^{2} \leqslant(\mu(x) / d)^{d}, \text { for } x \in K_{\mathrm{R}},
$$

the equality sign holding if and only if $|\sigma(x)|^{2}=|\tau(x)|^{2}$ for all $\mathbf{R}$-algebra homomorphisms $\sigma, \tau: K_{\mathbf{R}} \rightarrow \mathbf{C}$.

For $x \in R, x \neq 0$, we have $N(x)=|R / R x|$. The $\operatorname{ring} R$ is called Euclidean for the norm if for every $a, b \in R, b \neq 0$, there are $q, r \in R$ such that $a=q b+r$ and $N(r)<N(b)$. Using the multiplicativity of the norm one easily proves that $R$ is Euclidean for the norm if and only if for each $x \in K$ there exists $y \in R$ such that $N(x-y)<1$.

In the rest of this section we assume that every cube root of unity contained in $K$ is actually contained in $R$. This condition is necessary for $R$ to be Euclidean, since any unique factorization domain is integrally closed inside its field of fractions. Notice that the condition is satisfied if $K=\mathbf{Q}\left(\zeta_{m}\right)$ and $R=\mathbb{Z}\left[\zeta_{m}\right]$ for some integer $m \geqslant 1$.

(1.3) Lemma. Let $x \in K$ be such that $|\sigma(x)|^{2}=1$ and $|\sigma(x-u)|^{2}=1$ for some root of unity $u \in R$ and some field homomorphism $\sigma: K \rightarrow \mathbb{C}$. Then $x \in R$.

Proof. Let $y=\sigma\left(-x u^{-1}\right) \in \mathbf{C}$; then $y \bar{y}=1$ and $y+\bar{y}=-1$, so $y$ is a cube root of unity. Since $\sigma: K \rightarrow \mathbf{C}$ is injective, it follows that $-x u^{-1}$ is a cube root of unity in $K$. Therefore our assumption on $R$ implies that $-x u^{-1} \in R$; hence

$$
x=(-x u)^{-1} \cdot(-u) \in R .
$$

(1.4) Proposition. If $d$ is a usable bound for $F$, then $R$ is Euclidean for the norm.

Proof. Let $x \in K$ be arbitrary; we have to exhibit an element $y \in R$ for which $N(x-y)<1$. Using (1.1) we reduce to the case $x \in F$. Then $\mu(x) \leqslant d$, since $d$ is a bound for $F$. If the inequality is strict, then $N(x)<1$ by (1.2), and we can take $y=0$. If the equality sign holds, then $\mu(x)=\mu(x-u)=d$ for some root of unity $u \in R$, since $d$ is usable. We get

$$
\begin{gathered}
N(x)^{2} \leqslant(\mu(x) / d)^{d}=1, \\
N(x-u)^{2} \leqslant(\mu(x-u) / d)^{d}=1 .
\end{gathered}
$$

If at least one strict inequality holds, then we can take $y=0$ or $y=u$. If both equality signs hold, then

$$
|\sigma(x)|^{2}=|\tau(x)|^{2}, \quad|\sigma(x-u)|^{2}=|\tau(x-u)|^{2}
$$

for all $\sigma, \tau: K \rightarrow \mathbf{C}$, and since

$$
\begin{aligned}
& \prod_{\sigma}|\sigma(x)|^{2}=N(x)^{2}=1, \\
& \prod_{\sigma}|\sigma(x-u)|^{2}=N(x-u)^{2}=1
\end{aligned}
$$

it follows that $|\sigma(x)|^{2}=|\sigma(x-u)|^{2}=1$ for all $\sigma$. But then (1.3) asserts $x \in R$, contradicting $x \in F$ since $x \neq 0$. 


\section{Cyclotomic fields}

In the case when $K=\mathbb{Q}\left(\zeta_{m}\right)$ and $R=\mathbb{Z}\left[\zeta_{m}\right]$ for some integer $m \geqslant 1$, we write $\mu_{m}, F_{m}$ and $c_{m}$ instead of $\mu, F$ and $c$, respectively. The function $\operatorname{Tr}_{m}: \mathbf{Q}\left(\zeta_{m}\right)_{\mathbf{R}} \rightarrow \mathbb{R}$ denotes the natural extension of the trace $\mathbf{Q}\left(\zeta_{m}\right) \rightarrow \mathbf{Q}$. The field automorphism of $\mathbf{Q}\left(\zeta_{m}\right)$ which sends $\zeta_{m}$ to $\zeta_{m}{ }^{-1}$ extends naturally to an $\mathbf{R}$-algebra automorphism of $\mathbf{Q}\left(\zeta_{m}\right)_{\mathbf{R}}$, which is called complex conjugation and denoted by an overhead bar. For $x \in \mathbf{Q}\left(\zeta_{m}\right)_{\mathbf{R}}$, we have

$$
\mu_{m}(x)=\operatorname{Tr}_{m}(x \bar{x})
$$

Note that a similar formula holds for arbitrary $K$, if complex conjugation is suitably defined.

(2.2) Proposition. Let $n$ be a positive divisor of $m$, and

$$
e=\left[\mathbf{Q}\left(\zeta_{m}\right): \mathbf{Q}\left(\zeta_{n}\right)\right]=\phi(m) / \phi(n) .
$$

Then $c_{m} \leqslant e^{2} \cdot c_{n}$. Moreover, if $c^{\prime}$ is a usable bound for $F_{n}$, then $e^{2} . c^{\prime}$ is a usable bound for $F_{m}$.

The proof of (2.2) relies on the relative trace function $\mathbf{Q}\left(\zeta_{m}\right) \rightarrow \mathbf{Q}\left(\zeta_{n}\right)$ and its natural extension $\mathbf{Q}\left(\zeta_{m}\right)_{\mathbf{R}} \rightarrow \mathbf{Q}\left(\zeta_{n}\right)_{\mathbb{R}}$, notation: Tr. This is a $\mathbf{Q}\left(\zeta_{n}\right)_{\mathbf{R}}$-linear map, given by

$$
\operatorname{Tr}(x)=\sum_{\sigma \in G} \sigma(x), \text { for } x \in \mathbb{Q}\left(\zeta_{m}\right)_{\mathbf{R}},
$$

where $G$ denotes the Galois group of $\mathbf{Q}\left(\zeta_{m}\right)$ over $\mathbf{Q}\left(\zeta_{n}\right)$, acting naturally on $\mathbf{Q}\left(\zeta_{m}\right)_{\mathbf{R}}$. We have $\operatorname{Tr}_{m}=\operatorname{Tr}_{n} \circ \operatorname{Tr}$, and one easily proves that $\operatorname{Tr}$ commutes with complex conjugation.

(2.3) Lemma. Let $x \in \mathbf{Q}\left(\zeta_{m}\right)_{\mathbf{R}}$ and $y \in \mathbf{Q}\left(\zeta_{n}\right)_{\mathbf{R}}$. Then

$$
\mu_{m}(x)-\mu_{m}(x-y)=e\left(\mu_{n}\left(\frac{1}{e} \operatorname{Tr}(x)\right)-\mu_{n}\left(\frac{1}{e} \operatorname{Tr}(x)-y\right)\right) .
$$

Proof. Using (2.1), we find:

$$
\begin{aligned}
& e\left(\mu_{n}\left(\frac{1}{e} \operatorname{Tr}(x)\right)-\mu_{n}\left(\frac{1}{e} \operatorname{Tr}(x)-y\right)\right) \\
& \quad=e \cdot \operatorname{Tr}_{n}\left(\frac{1}{e} \operatorname{Tr}(x) \bar{y}+\frac{1}{e} \operatorname{Tr}(\bar{x}) y-y \bar{y}\right) \\
& \quad=\operatorname{Tr}_{n}(\operatorname{Tr}(x) \bar{y}+\operatorname{Tr}(\bar{x}) y-e \cdot y \bar{y}) \\
& =\operatorname{Tr}_{n}(\operatorname{Tr}(x \bar{y})+\operatorname{Tr}(\bar{x} y)-\operatorname{Tr}(y \bar{y})) \\
& =\operatorname{Tr}_{m}(x \bar{y}+\bar{x} y-y \bar{y}) \\
& =\mu_{m}(x)-\mu_{m}(x-y) .
\end{aligned}
$$

(2.4) Lemma. For $x \in \mathbf{Q}\left(\zeta_{m}\right)_{\mathbf{R}}$, we have

$$
\mu_{m}(x)=\frac{1}{m} \sum_{j=1}^{m} \mu_{n}\left(\operatorname{Tr}\left(x \zeta_{m}^{j}\right)\right) .
$$


Proof. In the computation below $\sum_{\sigma}$ and $\sum_{\tau}$ refer to summations over $G$.

$$
\begin{aligned}
\sum_{j=1}^{m} \mu_{n}\left(\operatorname{Tr}\left(x \zeta_{m}{ }^{j}\right)\right) & =\sum_{j=1}^{m} \mu_{n}\left(\sum_{\sigma} \sigma\left(x \zeta_{m}^{j}\right)\right) \\
& =\operatorname{Tr}_{n}\left(\sum_{j=1}^{m} \sum_{\sigma} \sum_{\tau} \sigma(x) \sigma\left(\zeta_{m}^{j}\right) \tau(\bar{x}) \tau\left(\zeta_{m}{ }^{-j}\right)\right) \\
& =\operatorname{Tr}_{n}\left(\sum_{\sigma} \sum_{\tau} \sigma(x) \tau(\bar{x})\left(\sum_{j=1}^{m}\left(\sigma\left(\zeta_{m}\right) \tau\left(\zeta_{m}\right)^{-1}\right)^{j}\right)\right)
\end{aligned}
$$

For $\sigma, \tau \in G$, let $\zeta_{\sigma, \tau}$ denote the $m$-th root of unity $\sigma\left(\zeta_{m}\right) \tau\left(\zeta_{m}\right)^{-1}$. Then $\zeta_{\sigma, \tau}=1$ if and only if $\sigma=\tau$, and

$$
\begin{aligned}
\sum_{j=1}^{m} \zeta_{\sigma, \tau}^{j} & =0 \quad \text { if } \quad \zeta_{\sigma, \tau} \neq 1 \\
& =m \quad \text { if } \quad \zeta_{\sigma, \tau}=1
\end{aligned}
$$

Hence the above expression becomes

$$
\operatorname{Tr}_{n}\left(\sum_{\sigma} \sigma(x) \sigma(\bar{x}) m\right)=m \cdot \operatorname{Tr}_{n}(\operatorname{Tr}(x \bar{x}))=m \cdot \operatorname{Tr}_{m}(x \bar{x})=m \cdot \mu_{m}(x) .
$$

This proves (2.4).

Proof of (2.2). Let $x \in F_{m}$; we have to prove $\mu_{m}(x) \leqslant e^{2} \cdot c_{n}$. Applying (2.3) with $y \in \mathbf{Z}\left[\zeta_{n}\right]$ we find that $x \in F_{m}$ implies $(1 / e) \operatorname{Tr}(x) \in F_{n}$. Since also $x \zeta_{m}{ }^{j}$ belongs to $F_{m}$, for $j \in \mathbb{Z}$, we have in the same way $(1 / e) \operatorname{Tr}\left(x \zeta_{m}^{j}\right) \in F_{n}$. Therefore

$$
\mu_{n}\left(\operatorname{Tr}\left(x \zeta_{m}^{j}\right)\right)=e^{2} \cdot \mu_{n}\left(\frac{1}{e} \operatorname{Tr}\left(x \zeta_{m}^{j}\right)\right) \leqslant e^{2} \cdot c_{n}
$$

for all $j \in \mathbf{Z}$, and (2.4) implies that $\mu_{m}(x) \leqslant e^{2} \cdot c_{n}$. This proves that $c_{m} \leqslant e^{2} \cdot c_{n}$. Next assume that $c^{\prime}$ is a usable bound for $F_{n}$, and let $x \in F_{m} \cap \mathbf{Q}\left(\zeta_{m}\right)$ satisfy $\mu_{m}(x)=e^{2} \cdot c^{\prime}$. Then the above reasoning implies that $c^{\prime}=c_{n}$ and

$$
\mu_{n}\left(\frac{1}{e} \operatorname{Tr}\left(x \zeta_{m}^{j}\right)\right)=c_{n}=c^{\prime} \quad \text { for all } j \in \mathbf{Z} .
$$

Taking $j=0$ we find that $(1 / e) \operatorname{Tr}(x)$ is an element of $F_{n} \cap \mathbf{Q}\left(\zeta_{n}\right)$ for which

$$
\mu_{n}\left(\frac{1}{e} \operatorname{Tr}(x)\right)=c^{\prime} .
$$

Since $c^{\prime}$ is a usable bound for $F_{n}$, there is a root of unity $u \in \mathbf{Z}\left[\zeta_{n}\right]$ such that

$$
\mu_{n}\left(\frac{1}{e} \operatorname{Tr}(x)-u\right)=c^{\prime} .
$$

Applying (2.3) with $y=u$ we get $\mu_{m}(x-u)=\mu_{m}(x)=e^{2} \cdot c^{\prime}$, which proves that $e^{2} \cdot c^{\prime}$ is a usable bound for $F_{m}$.

Without proof we remark that the equality sign holds in (2.2) if $m$ and $n$ are divisible by the same primes.

Since $c_{1}=\frac{1}{4}$ is a usable bound for $F_{1}$, we conclude from $(2.2)$ that $\frac{1}{4} \phi(m)^{2}$ is a usable bound for $F_{m}$, for any $m$. If $\phi(m) \leqslant 4$, then it follows that $\phi(m)$ is a usable 
bound for $F_{m}$, and that $\mathbf{Z}\left[\zeta_{m}\right]$ is Euclidean for the norm, by (1.4). This gives us exactly the cases $m=1,3,4,5,8,12$ which were already known. In $\$ 4$ we will obtain better results by applying (2.2) to a prime divisor $n$ of $m$.

\section{A computation in linear algebra}

Let $n \geqslant 2$ be an integer, and let $V$ be an $(n-1)$-dimensional $\mathbf{R}$-vector space with generators $e_{i}, 1 \leqslant i \leqslant n$, subject only to the relation $\sum_{i=1}^{n} e_{i}=0$. The positive definite quadratic form $q$ on $V$ is defined by

$$
q(x)=\sum_{1 \leqslant i<j \leqslant n}\left(x_{i}-x_{j}\right)^{2}, \text { for } x=\sum_{i=1}^{n} x_{i} e_{i} \in V .
$$

Denote by $():, V \times V \rightarrow \mathbf{R}$ the symmetric bilinear form induced by $q$ :

Then

$$
(x, y)=\frac{1}{2}(q(x+y)-q(x)-q(y)) .
$$

$$
\begin{array}{ll}
(x, x)=q(x), & \text { for } \quad x \in V, \\
\left(e_{i}, e_{i}\right)=n-1, & \text { for } \quad 1 \leqslant i \leqslant n, \\
\left(e_{i}, e_{j}\right)=-1, & \text { for } \quad 1 \leqslant i<j \leqslant n .
\end{array}
$$

The subgroup $L$ of $V$ generated by $\left\{e_{i} \mid 1 \leqslant i \leqslant n\right\}$. is a lattice of rank $n-1$ in $V$. The fundamental domain

$$
\begin{aligned}
E & =\{x \in V \mid q(x) \leqslant q(x-y) \text { for all } y \in L\} \\
& =\left\{x \in V \mid(x, y) \leqslant \frac{1}{2} q(y) \text { for all } y \in L\right\}
\end{aligned}
$$

is a compact subset of $V$, and we put

$$
b=\max \{q(x) \mid x \in E\} .
$$

(3.1) Proposition. The set of points $x \in E$ for which $q(x)=b$ is given by

$$
\left\{\frac{1}{n} \sum_{i=1}^{n} i e_{\sigma(i)} \mid \sigma \text { is a permutation of }\{1,2, \ldots, n\}\right\} \text {. }
$$

Moreover,

$$
b=\frac{n^{2}-1}{12} .
$$

This proposition is proved after a series of lemmas. We put $N=\{1,2, \ldots, n\}$. For $A \subset N$, let $e_{A}=\sum_{i \in A} e_{i}$. We call $A$ proper if $\varnothing \neq A \neq N$.

(3.3) Lemma. Let $y \in L$ be such that $y \neq e_{A}$ for all $A \subset N$. Then there is an element $z= \pm e_{j} \in L$ such that

$$
q(z)+q(y-z)<q(y) .
$$

Proof. Let $y=\sum_{i=1}^{n} m_{i} e_{i}$ with $m_{i} \in \mathbf{Z}$. Using $\sum_{i=1}^{n} e_{i}=0$ we may assume that $0 \leqslant \sum_{i=1}^{n} m_{i} \leqslant n-1$. For $z= \pm e_{j}$ we have

$$
\begin{aligned}
\frac{1}{2}(q(y)-q(z)-q(y-z)) & =(y, z)-(z, z) \\
& = \pm\left(n m_{j}-\sum_{i=1}^{n} m_{i}\right)-(n-1) .
\end{aligned}
$$


If this is $>0$ for some $j$ and some choice of the sign we are done. Therefore suppose it is $\leqslant 0$ for all $j$ and for both signs. Then for $1 \leqslant j \leqslant n$ we have

$$
\begin{aligned}
& n m_{j} \leqslant\left(\sum_{i=1}^{n} m_{i}\right)+(n-1) \leqslant 2 n-2<2 n, \\
& n m_{j} \geqslant\left(\sum_{i=1}^{n} m_{i}\right)-(n-1) \geqslant-n+1>-n,
\end{aligned}
$$

so $m_{j} \in\{0,1\}$ for all $j$. Hence $y=e_{A}$ for some $A \subset N$, contradicting our assumption.

(3.4) LeMMA. Let $x \in V$. Then $x \in E$ if and only if $\left(x, e_{A}\right) \leqslant \frac{1}{2} q\left(e_{A}\right)$ for all $A \subset N$.

Proof. The "only if" part is clear. "If": we know that

$$
\left(x, e_{A}\right) \leqslant \frac{1}{2} q\left(e_{A}\right) \text { for all } A \subset N
$$

and we have to prove that

$$
(x, y) \leqslant \frac{1}{2} q(y) \text { for all } y \in L .
$$

This is done by an obvious induction on $q(y)$, using (3.3).

(3.5) LeMma. Let $x_{0} \in E$ satisfy $q\left(x_{0}\right)=b$. Then there are $n-1$ different proper subsets $A(i) \subset N$, for $1 \leqslant i \leqslant n-1$, such that $x_{0}$ is the unique solution of the system of linear equations

$$
\left(x, e_{A(i)}\right)=\frac{1}{2} q\left(e_{A(i)}\right), \quad 1 \leqslant i \leqslant n-1 .
$$

Proof. Put

$$
S=\left\{A \subset N \mid\left(x_{0}, e_{A}\right)=\frac{1}{2} q\left(e_{A}\right)\right\},
$$

then $\left(x_{0}, e_{A}\right)<\frac{1}{2} q\left(e_{A}\right)$ for each $A \subset N, A \notin S$. If the linear span of $\left\{e_{A} \mid A \in S\right\}$ has dimension $n-1$, then there are $n-1$ subsets $A(i) \in S$ such that $\left\{e_{A(i)} \mid 1 \leqslant i \leqslant n-1\right\}$ is linearly independent over $\mathbb{R}$. Then clearly $x_{0}$ is the unique solution of (3.6), and each $A(i)$ is proper since $e_{A(i)} \neq 0$.

Therefore suppose that the linear span of $\left\{e_{A} \mid A \in S\right\}$ has codimension $\geqslant 1$ in $V$. Then for some $z \in V, z \neq 0$, we have

$$
\left(z, e_{A}\right)=0 \text { for all } A \in S .
$$

Multiplying $z$ by a suitably chosen real number we can achieve that

$$
\begin{aligned}
& \left(x_{0}, z\right) \geqslant 0 \\
& \left(z, e_{A}\right) \leqslant \frac{1}{2} q\left(e_{A}\right)-\left(x_{0}, e_{A}\right) \text { for all } A \subset N, \quad A \notin S .
\end{aligned}
$$

Then for all $A \subset N$ we have $\left(x_{0}+z, e_{A}\right) \leqslant \frac{1}{2} q\left(e_{A}\right)$, which implies $x_{0}+z \in E$, by (3.4). But using (3.7) we find that

$$
q\left(x_{0}+z\right) \geqslant q\left(x_{0}\right)+q(z)>q\left(x_{0}\right),
$$

which contradicts our assumption $q\left(x_{0}\right)=b=\max \{q(x) \mid x \in E\}$.

(3.8) Lemma. Let $x_{0} \in E$, and let $A, B \subset N$ be such that

$$
\left(x_{0}, e_{A}\right)=\frac{1}{2} q\left(e_{A}\right), \quad\left(x_{0}, e_{B}\right)=\frac{1}{2} q\left(e_{B}\right) .
$$

Then $A \subset B$ or $B \subset A$. 
Proof. Put $C=A-B$ and $D=B-A$. If $C=\varnothing$ or $D=\varnothing$ we are done, so suppose $C \neq \varnothing \neq D$. Then $C \cap D=\varnothing$ implies

$$
\left(e_{A \cap B}, e_{A \cup B}\right)-\left(e_{A}, e_{B}\right)=-\left(e_{C}, e_{D}\right)=|C| \cdot|D|>0 \text {. }
$$

Using $e_{A \cap B}+e_{A \cup B}=e_{A}+e_{B}$ we find that

$$
\begin{aligned}
\left(x_{0}, e_{A \cap B}\right)+\left(x_{0}, e_{A \cup B}\right) & =\left(x_{0}, e_{A}\right)+\left(x_{0}, e_{B}\right) \\
& =\frac{1}{2} q\left(e_{A}\right)+\frac{1}{2} q\left(e_{B}\right) \\
& =\frac{1}{2} q\left(e_{A}+e_{B}\right)-\left(e_{A}, e_{B}\right) \\
& >\frac{1}{2} q\left(e_{A \cap B}+e_{A \cup B}\right)-\left(e_{A \cap B}, e_{A \cup B}\right) \\
& =\frac{1}{2} q\left(e_{A \cap B}\right)+\frac{1}{2} q\left(e_{A \cup B}\right) .
\end{aligned}
$$

Hence for $X=A \cap B$ or for $X=A \cup B$ we have $\left(x_{0}, e_{X}\right)>\frac{1}{2} q\left(e_{X}\right)$, contradicting $x_{0} \in E$.

Proof of (3.1). Let $x_{0} \in E$ satisfy $q\left(x_{0}\right)=b$, and let $\{A(i) \mid 1 \leqslant i \leqslant n-1\}$ be a system of $n-1$ proper subsets of $N$ as in (3.5). By (3.8), this system is linearly ordered by inclusion. This is only possible if after a suitable renumbering of the vectors $e_{i}$ and the sets $A(i)$ we have

$$
A(i)=\{i+1, i+2, \ldots, n\}, \text { for } 1 \leqslant i \leqslant n-1 .
$$

By (3.5) we have

$$
\sum_{j=i+1}^{n}\left(x_{0}, e_{j}\right)=\frac{1}{2} q\left(e_{A(i)}\right)=\frac{1}{2} i(n-i), \text { for } \quad 1 \leqslant i \leqslant n-1 .
$$

Write $x_{0}=\sum_{j=1}^{n} x_{j} e_{j}$ in such a manner that $\sum_{j=1}^{n} x_{j}=0$. Then $\left(x_{0}, e_{j}\right)=n x_{j}$; so our system becomes

$$
\sum_{j=i+1}^{n} n x_{j}=\frac{1}{2} i(n-i), \text { for } 0 \leqslant i \leqslant n-1 .
$$

This implies

$$
\begin{gathered}
n x_{i}=i-\frac{1}{2}(n+1), \text { for } 1 \leqslant i \leqslant n, \\
x_{0}=\frac{1}{n} \sum_{i=1}^{n} i e_{i} .
\end{gathered}
$$

We renumbered the $e_{i}$ once; so we conclude that $x_{0}$ is in the set (3.2). Since at least one $x_{0} \in E$ satisfies $q\left(x_{0}\right)=b$, it follows for reasons of symmetry that conversely every element $x$ of (3.2) satisfies $x \in E$ and $q(x)=b$. Finally,

$$
b=\sum_{1 \leqslant i<j \leqslant n}(i-j)^{2} / n^{2}=\left(n^{2}-1\right) / 12 .
$$

This proves (3.1).

\section{Proof of the theorem}

(4.1) Proposition. Let $n$ be a prime number. Then $c_{n}=\left(n^{2}-1\right) / 12$, and this is a usable bound for $F_{n}$.

Proof. We apply the results of $\S 3$. The $\mathbf{R}$-vector space $\mathbf{Q}\left(\zeta_{n}\right)_{\mathbf{R}}$ is generated by $n$ elements $\zeta_{n}{ }^{i}, 1 \leqslant i \leqslant n$, subject only to the relation $\sum_{i=1}^{n} \zeta_{n}{ }^{i}=0$. For real numbers 
$x_{i}, 1 \leqslant i \leqslant n$, we have

$$
\begin{aligned}
\mu_{n}\left(\sum_{i=1}^{n} x_{i} \zeta_{n}{ }^{i}\right) & =\operatorname{Tr}_{n}\left(\sum_{i=1}^{n} \sum_{j=1}^{n} x_{i} x_{j} \zeta_{n}^{i-j}\right) \\
& =n \cdot \sum_{i=1}^{n} x_{i}{ }^{2}-\sum_{i=1}^{n} \sum_{j=1}^{n} x_{i} x_{j} \\
& =\sum_{1 \leqslant i<j \leqslant n}\left(x_{i}-x_{j}\right)^{2} .
\end{aligned}
$$

Therefore there is an isomorphism of quadratic spaces $\left(\mathbf{Q}\left(\zeta_{n}\right)_{\mathbf{R}}, \mu_{n}\right) \cong(V, q)$ which maps $\zeta_{n}{ }^{i}$ to $e_{i}$, for $1 \leqslant i \leqslant n$. Clearly, $\mathbb{Z}\left[\zeta_{n}\right]$ corresponds to $L$, so $F_{n}$ corresponds to $E$ and $c_{n}=b$. Translating (3.1) we find: $c_{n}=\left(n^{2}-1\right) / 12$, and the set of $x \in F_{n}$ for which $\mu_{n}(x)=c_{n}$ is given by

$$
\left\{\frac{1}{n} \sum_{i=1}^{n} i \zeta_{n}{ }^{\sigma(i)} \mid \sigma \text { is a permutation of }\{1,2, \ldots, n\}\right\} \text {. }
$$

Let $x$ be in this set. Putting $\sigma(0)=\sigma(n)$ we have

$$
x-\zeta_{n}{ }^{\sigma(n)}=\frac{1}{n} \sum_{i=0}^{n-1} i \zeta_{n}{ }^{\sigma(i)}=\frac{1}{n} \sum_{j=1}^{n} j \zeta_{n}{ }^{\sigma(j-1)} .
$$

This element belongs to the set (4.2), so $\mu_{n}\left(x-\zeta_{n}{ }^{\sigma(n)}\right)=c_{n}$, which proves usability of $c_{n}$.

We turn to the proof of the theorem. The cases $m=1,3,4,5,8,12$ have been dealt with in $\$ 2$. Further, (2.2) and (4.1) imply that

$$
\begin{aligned}
c_{7} & =4<6=\phi(7), \\
c_{9} & \leqslant 3^{2} \cdot c_{3}=6=\phi(9), \\
c_{11} & =10=\phi(11), \\
c_{15} & \leqslant 2^{2} \cdot c_{5}=8=\phi(15), \\
c_{20} & \leqslant 2^{2} \cdot c_{5}=8=\phi(20),
\end{aligned}
$$

and in each of these cases $\phi(m)$ is a usable bound for $F_{m}$. Application of (1.4) concludes the proof.

Without proof we remark that our method does not apply to other fully cyclotomic fields:

(4.3) Proposition. Let $m \geqslant 1$ be an integer for which $c_{m} \leqslant \phi(m)$. Then $\phi(m) \leqslant 10$ and $m \neq 16, m \neq 24$.

\section{References}

1. J. W. S. Cassels, "On a conjecture of R. M. Robinson about sums of roots of unity", J. Reine Angew. Math., 238 (1969), 112-131.

2. C. F. Gauss, Werke, Zweiter Band (Göttingen, 1876).

3. G. H. Hardy and E. M. Wright, An introduction to the theory of numbers (Oxford, 1938).

4. R. B. Lakein, "Euclid's algorithm in complex quartic fields", Acta Arith., 20 (1972), 393-400.

5. E. Landau, Vorlesungen über Zahlentheorie, Band 3 (Leipzig, 1927).

6. J. M. Masley, On the class number of cyclotomic fields (thesis, Princeton University, 1972). 
7. _- "On cyclotomic fields Euclidean for the norm map", Notices Amer. Math. Soc., 19 (1972), A-813 (abstract 700-A3).

8. J. Ouspensky, "Note sur les nombres entiers dépendant d'une racine cinquième de l'unité ", Math. Ann., 66 (1909), 109-112.

9. P. J. Weinberger, "On Euclidean rings of algebraic integers", Proc. Symp. Pure Math., 24 (Analytic Number Theory), 321-332 (Amer. Math. Soc., 1973).

Mathematisch Instituut,

Universiteit van Amsterdam,

Amsterdam, The Netherlands. 
